\title{
SATURATING ABILITY OF THE BORAX MELT
}

\author{
L.I. Fedorenkova*, G.P. Fedina \\ Oles Honchar Dnipro National University, Dnipro, Ukraine \\ e-mail:Luba.Fed@gmail.com
}

The work is devoted to the research of influence of the electrolysis on the saturating ability of the electrolyte, which is a borax melt. To establish regularity when the saturating medium activity changes, which influences the intensification of saturation process, the structure and properties of borax during electrolysis were investigated. The change in the saturating medium activity was estimated by a relative value equal to the ratio of the boride layer depth obtained by non-electrolysis borating to the layer depth obtained by electrolysis borating. When comparing the real and theoretical saturating abilities, their convergence was observed. It was found that the saturation ability of the borax melt increases with increasing electrolyte runtime, which is due to a change in the structural components of the borax melt. A quantum-chemical calculation of the borax molecule structure was carried out using the non-empirical method of molecular orbitals in the theory of the density functional (DFT) with the three-parameter exchange-correlation functional B3LYP. Calculations show that two main structures are characteristic of a borax molecule: noncoplanar, with two mutually perpendicular 4-membered boron-oxygen cycles and flat linear. An increase in the saturation ability of the borax melt during electrolysis allows the development of new low-energy borating processes.

Keywords: borax melt, non-electrolysis borating, equilibrium concentration, saturating ability of electrolyte, polarization orbitals, density functional method.

Received 09.11.2019; Received in revised form 10.12.2019; Accepted 23.12.2019

\section{Introduction}

The hardening of metals and alloys by creating a high-strength boride layer on their surface is carried out using CTT (chemico-thermal treatment) in the molten electrolyte, the main component of which is a mineral of borax or sodium tetraborate [1]. During the electrolysis, the borax structure is undergoing changes, which affect the saturation ability of the electrolyte. The saturating ability is directly dependent on the saturating medium activity, which, according to the definition of Yu. P. Lakhtin [3] is characterized by the equilibrium concentration of the diffusing element. Maximum electrolyte activity according to [2] is observed after working out the electrolyte for 40-60 hour since the melting of the borax. This allows to develop new low-energy borating processes, one of which is liquid borating (nonelectrolysis). To establish regularity when the saturating medium activity changes, which influences on the intensification of the saturation process, the structure and properties of borax during electrolysis were investigated.

\section{Materials and methods}

The samples of steel 20 was carried out in the borax melt at a current density of 0,1-1,25 $\mathrm{A} / \mathrm{cm}^{2}$, where the cathode is samples and the anode is graphite rods. The borating included several stages:

Stage I - liquid non-electrolysis borating at a current strength in the crucible - anode circuit 200A and a temperature of $1446 \mathrm{~K}$ in a freshly prepared borax melt. Samples were hung in 2 hours after unloading the previous one. Every three hours of bath work, borax samples were taken.

Stage II - borating was carried out in an electrolyte that worked for many hours. Samples were subjected to electrolysis borating for 1 hour. A borax sample was taken before each sample treatment.

Stage III - borating in the electrolyte after many hours work with replenishment of fresh borax and many times cooling and heating of the melt. The borax samples were hung together with the details electrolyzed, without connecting to the electrolysis circuit. 
To study structural changes in the borax, its normalized volumes were solidified with different cooling rates $V_{1}>V_{2}>V_{2}$. Structural changes in the borax melt were determined using chemical analysis and X-ray diffraction analysis on a DRON-2 diffractometer in Co radiation.

Kinetics of diffusion zone growth in saturating media with different activity and melt state was investigated using metallographic analysis with NEOPHOT 21 microscope and spectral analysis according to the method [7].

To perform a quantum chemical calculation of the borax molecule structure, the nonempirical molecular orbitals method in the density functional theory (DFT) with the three-parameter exchange-correlation functional B3LYP was used [4-5].

The geometric parameters of molecular structures were optimized using a valencesplit basis G311 ++ G (3df), which includes two diffusion and four polarization orbitals for oxygen, boron, and sodium atoms. Atom charges and atomic spin populations were calculated based on the Mulliken electron density. The calculation results for each structure were presented in the classical form of geometric parameters (III) and in the form of filling the molecule with electron density (III).

\section{Results and discussion}

The results of chemical analysis and X-ray diffraction analysis of the melt samples at the second stage of the studies showed the boron concentration in the melt increases from $21.3 \mathrm{wt} . \%$ to $21.6 \mathrm{wt} . \%$. According to metallographic analysis, the saturation ability of the electrolyte affects the depth of the forming boride layer and increases with increasing electrolyte run time. The dependence of the boride layer thickness on activation time of the melt by electric current for both treatment types is shown in Fig. 1.

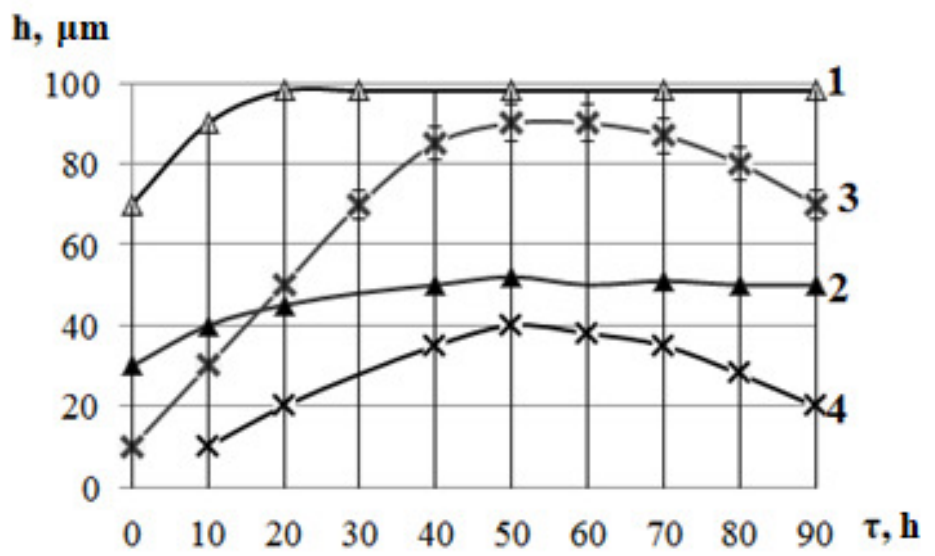

Fig. 1. Dependence of the two-phase boride layer thickness obtained on samples at: 1,2 - electrolysis borating; 3, 4 - non-electrolysis borating $(1,3$ - total layer thickness; 2, 4 - thickness of monoboride $\mathrm{FeB}$ ) from the time of melt activation.

It can be seen from the Fig. $1(1,2)$ the kinetics of the boride layer formation during electrolysis borating is described by a parabolic dependence for both diboride $\mathrm{Fe}_{2} \mathrm{~B}$ and monoboride FeB. For samples boronized without electrolysis, saturation activity maxima occurring in 50-60 hours of melt activation by current are observed.

According to [3], the saturation ability is directly dependent on saturation activity and is characterized by the equilibrium concentration of the diffusing element. To find the equilibrium concentration depending on the variable parameters of mathematical modeling method based on the diffusing element flow through the section surface of metal - saturation medium was used: 


$$
D \frac{\partial C(0, \tau)}{\partial x}=\beta\left[C^{0}(\tau)-C(0, \tau)\right]
$$

$C(0, \tau)$ - diffusing element concentration on the metal surface; $C^{0}(\tau)=C_{n}-$ equilibrium concentration of the diffusing element, proportional to activity or saturation potential;

$$
\beta=\frac{\delta}{2 \tau} \ln \frac{C_{n}-\bar{C}}{C_{n}-C_{0}}
$$

- mass transfer coefficient, $\mathrm{m} / \mathrm{s} ; C_{0}-$ initial concentration of the diffusing element in the metal; $\bar{C}=\frac{C_{01}+C_{0 n}}{2}-$ final average element concentration in the diffusion layer; $n-$ number of steps to split; $x=\delta$ - saturation depth, $\mathrm{m} ; \tau$ - saturation time, h;

$$
D=\frac{x_{\tau}^{2}}{k \tau} .
$$

- diffusion coefficient, $\mathrm{m}^{2} / \mathrm{s} ; k=1,1 \cdot 10^{-6} \mathrm{sm} / \mathrm{s}$ - parabolic growth constant. With regard to (2) and (3) the expression (1) will have the form:

$$
\frac{\partial C(0, \tau)}{\partial x} \frac{1}{C_{n}-C(0, \tau)}=\frac{k}{2 x} \ln \frac{C_{n}-\bar{C}}{C_{n}-C_{0}} .
$$

The solution of the differential equation is presented in the form:

$$
\frac{C_{n}-C(0, \tau)}{C_{n}-\bar{C}}=\frac{x^{-k / 2}}{C_{n}-C_{0}}
$$

where $C(0, \tau)$ varies from 16 to $25 \%$ with step 0,$5 ; C_{0}$ - ranging from 0,1 to $8,9 \%$ and from 8,9 to $16,4 \%$ with step 0,$4 ; x$ varies from 0 to $100 \mu \mathrm{m}$ with step 10 . The calculation results carried out using MathCard are presented in Fig. 2. It is seen from the Fig. 2 an equilibrium concentration is directly dependent on concentration of the diffusing element in the melt and in the outer layers of the saturated surface.

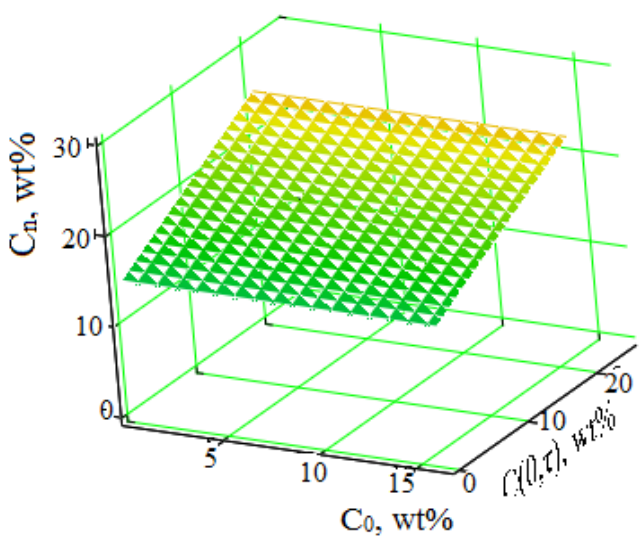

Fig. 2. Change in the equilibrium concentration depending on diffusing element concentration on the metal surface $\mathrm{C}(0, \tau)$ and initial concentration of the diffusing element in the metal $\mathrm{C}_{0}$. 
Thus, as the boron concentration on the surface increases, the saturating medium activity increases. Based on the calculations, the theoretical electrolyte activity depending on the boron concentration can be estimated at 0,59. When compared with the experimental indicator of the saturating medium activity, their convergence was observed.

According to metallographic analysis a non-electrolysis borating carried out: a without the addition of fresh portions of borax, $b$ - with replenishment with fresh borax, occurs under conditions of periodic changes in the saturating activity (Fig. 3). Actual saturation activity was estimated by a relative value equal to the ratio of the layer depth obtained under non-electrolysis borating to the layer depth obtained by electrolysis borating.

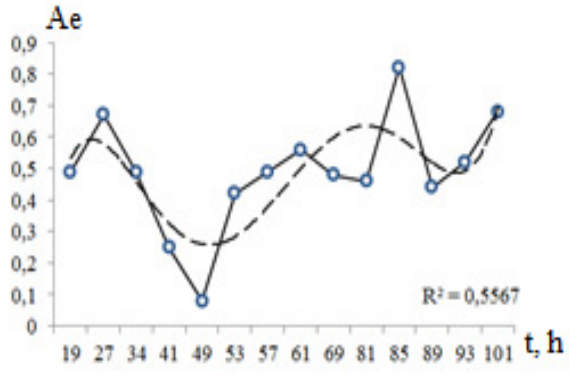

a)

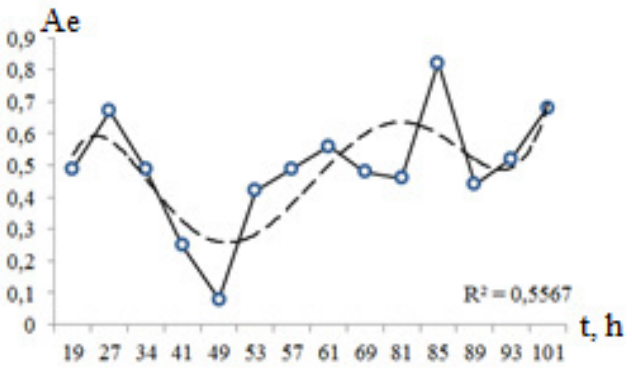

b)

Fig. 3. Dependence of the actual saturation activity $\left(A_{e}\right)$ on the activation time of the melt by current with polynomial approximation with degree 5 for case a) and degree 6 for case $b$ ).

The reliability of the approximation for each case is indicated on the graphs.

The oscillation of the actual saturation activity $\left(\mathrm{A}_{\mathrm{e}}\right)$ is determined by the borax melt state. It was not possible to establish the regularity of these changes. However, taking into account changes in the structure of the borax during electrolysis, the observed increase in activity can be associated with the formation of crystalline hydrate modifications, as well as the appearance of a boron-containing compound, the decomposition of which is accompanied by the release of active boron.

According to X-ray diffraction analysis of borax samples taken at different cooling rates and duration of electrolyte operation, crystalline regions of anhydrous borax were found in the borax structure. It has been established that the borax samples composition after working out the electrolyte mainly includes five-water borax, anhydrous borax $\left(\mathrm{Na}_{2} \mathrm{~B}_{4} \mathrm{O}_{7}\right)$ and $\mathrm{FeB}$. According to $[8,9]$, the borax structure is considered as a network of boron-oxygen rings to which sodium and hydroxyl groups are attached.

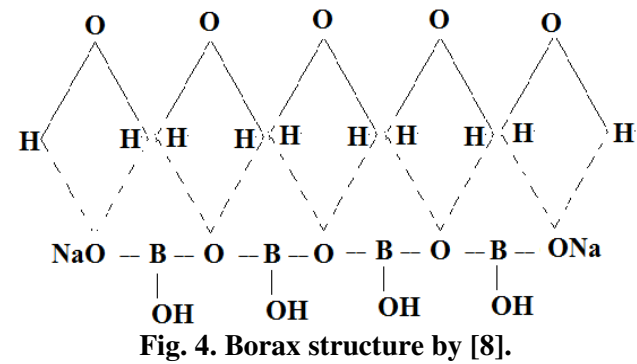

Water is an important link in boron structures; it often determines the very existence of a crystal lattice and provides it with spatial bonds through hydrogen. The water loss leads to the destruction of the crystal lattice. Easy formation of oxygen bonds, the flat 
structure of $\mathrm{BO}_{3}{ }^{-3}$, together with uneven valency, is the cause of glassy forms in borates (endless chains). Water is in the hydroxyls form and molecules in the borax structure. In this case, hydroxyl bonds included in the composition of borates - ions, are much stronger than the bonds of water molecules with a cation. This causes a difference in their destruction temperatures. So, according to $[9,10,11]$, dehydration at a temperature of $298 \mathrm{~K}$ with a loss 6,6 moles of water does not lead to a change in the borax structure, and at a temperature of $333 \mathrm{~K}$ with a loss 6,6 moles of water it leads to the five-water borax formation. This structure is hygroscopic, easily gathering water. Dehydration at $353 \mathrm{~K}$ with a loss 6,6 moles of water leads to amorphous dihydride formation. With further dehydration the borax turns into monohydride $(303 \mathrm{~K})$, then the borax is completely dehydrated, losing crystalline and constitutional water at a temperature of $623 \mathrm{~K}$. At the melting temperature, hydrogen bonds break, which leads to the crystal lattice destruction and the amorphous differences formation. Melt temperature increase and the addition of metal oxides in it leads to the disaggregation of boron complexes, to increase its fluidity and improve electrical conductivity. It is believed that borax in a liquid state dissociates into boric anhydride and sodium metaborate [10] and the effectiveness of borax as a flux dissolving metal oxides is due precisely to this process of thermal dissociation [9]. The temperature ranges of electrolysis borating $(1073-1273 \mathrm{~K})$ is much lower than the evaporation temperature of tetraborate $(1473 \mathrm{~K})$; therefore, the stability of the $\mathrm{Na}_{2} \mathrm{~B}_{4} \mathrm{O}_{7}$ compound in borate electrolytes is very large and its thermal dissociation to $\mathrm{Na}_{2} \mathrm{O}+2 \mathrm{~B}_{2} \mathrm{O}_{3}$ or $2 \mathrm{NaBO}_{2}+\mathrm{B}_{2} \mathrm{O}_{3}$ is fundamentally possible. According to quantum chemical calculations, the borax molecule is characterized by two main structures: non-coplanar, with two mutually perpendicular 4-membered boron-oxygen cycles and flat linear (structure I) (Fig. 5).

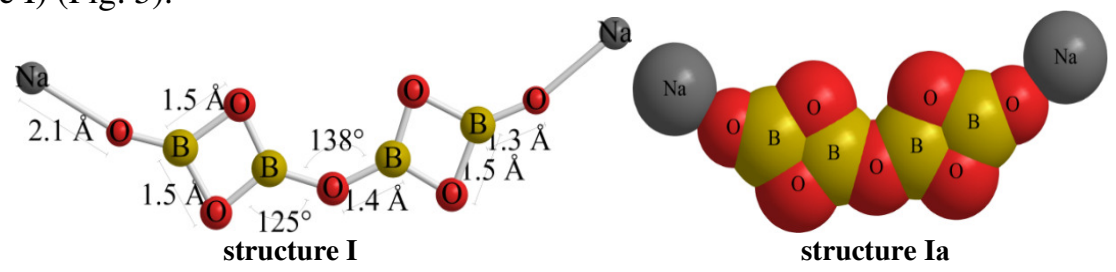

Fig.5. Flat linear borax structure. Spatial fill model.

In both cases, these structures can be considered as ethers of the diboric acid $\mathrm{H}_{2} \mathrm{~B}_{2} \mathrm{O}_{4}$ and its sodium salts. The angle formed by boron atoms and the ether oxygen atom for a non-coplanar structure is $98^{\circ}$, and for a similar characteristic of structure I is $138^{\circ}$. These angles deviate significantly from the equilibrium angle between the bonds formed by the oxygen atom in the water molecule (II) (Fig. 6).

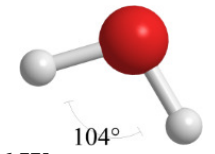

Fig. 6 Water structure (II).

Due to the tension of the considered bond angles in both borax structures during electrolysis, after cleavage of sodium ions, decomposition into 4 times ionized diborate particles takes place (structure III) (Fig. 7).

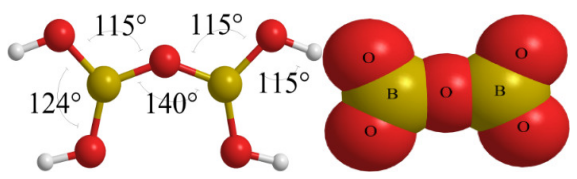

Fig. 7. Ionized diborate particles (Structure III). 
Particles of structure III have a higher symmetry and, due to mutual repulsion between two oxygen pairs (and inside each pair), the ether angle B-O-B increases up to $180^{\circ}$, becoming extremely stressed. Such structure promotes an increase in the boron concentration and, thereby, increases the activity of the saturating medium during CTT in the borax melt. Thus, the diborate particle structure obtained by the calculated method is consistent with experimental data on the structural state of borax samples taken during electrolysis.

\section{Conclusions}

Thus, according to research of saturating state influence on the intensification of saturation process during CTT in the borax melt, the following regularities are established:

1) the saturating ability during CTT in the borax melt depends on the equilibrium boron concentration at the section surface of metal - saturation medium and is directly dependent on the boron concentration on the hardened sample surface;

2) increasing the saturating ability of the electrolyte is due to a change in the structural components of the borax melt during electrolysis;

3) when comparing the real and theoretical saturating ability, their convergence is observed;

4) quantum chemical calculations show that the borax molecule is characterized by two main structures: non-coplanar, with two mutually perpendicular 4-membered boronoxygen cycles and a flat linear. Borax molecule splits sequentially during electrolysis borating to $\mathrm{O}^{2-}, \mathrm{BO}^{2-}$ and $\mathrm{B}^{3+}$ ions. Ionized particles of diborate (structure III) formed during electrolysis affect the activation of diffusion processes, promoting to its growth due to the formation of free boron ions;

increasing the saturation ability of borax during electrolysis allows the development of new low-energy borating.

\section{References}

1. Lyakhovich, L.S. and others. Progressivnyye metody termicheskoy i khimikotermicheskoy obrabotki. M: Mashinostroyeniye, 1972. - P.145-151.

2. Fedorenkova, L. Sposib ridynnoho boruvannya stalevykh vyrobiv / L. Fedorenkova, V.I. Mostovoi, V.D. Kolyuchaya // Patent na korysnu model' UA 96419. 10.02.2015. - №3.

3. Lakhtin, Yu.M., Arzamasov B.N. KHTO metallov. - M: Metallurgiya. - 1980. - 140 p.

4. Soloviev M.E., Soloviev M. M.// Komp'yuternaya khimiya. - M. - Salon-Press, 2005. - P. 390-416.

5. Bakulov V. A., Marghenin Yu. Yu., Subotina Yu. L. Kvantovo-khimicheskiy raschet organicheskikh molekul: Yekaterinburg. - GOU VPO UGTU-UPI, 2005. - 28 p.

6. Kobzev V. I. Primeneniye neempiricheskikh i poluempiricheskikh metodov v kvantovo-khimicheskikh raschetakh: Orenburg. - GOU OGU, 2004. - 150 p.

7. Tverdokhlebova S.V., Spiridonova I.M., Bondarenko A.M. // Zavodskaya laboratoriya. - 1990. - №11. - P. 46.

8. Boraty i boratnyye sistemy. Pod red. Slaydin G.R., Troitskaya N.V., Godet G.K.1978. - $142 \mathrm{p}$.

9. Godet G. K. Sintez boratov. AN Latv. SSR, Riga. $-1980 .-320$ p.

10. Nikolaev A.V. Fiziko-khimicheskoye izucheniye prirodnykh boratov. Riga: AN Latv. SSR, 1947. -53 p. 